DOI 10.4171/JEMS/212

David Eisenbud · Frank-Olaf Schreyer

\title{
Cohomology of coherent sheaves and series of supernatural bundles
}

Received February 10, 2009 and in revised form September 13, 2009

\begin{abstract}
We show that the cohomology table of any coherent sheaf on projective space is a convergent—but possibly infinite—sum of positive real multiples of the cohomology tables of what we call supernatural sheaves.
\end{abstract}

\section{Introduction}

Let $\mathbb{K}$ be a field, and let $\mathcal{F}$ be a coherent sheaf on $\mathbb{P}^{n}=\mathbb{P}_{\mathbb{K}}^{n}$. The cohomology table of $\mathcal{F}$ is the collection of numbers

$$
\gamma(\mathcal{F})=\left(\gamma_{i, d}\right) \quad \text { with } \quad \gamma_{i, d}=\operatorname{dim} H^{i}\left(\mathbb{P}^{n}, \mathcal{F}(d)\right),
$$

which we think of as an element of the real vector space $\prod_{d=-\infty}^{\infty} \mathbb{R}^{n+1}$.

In Eisenbud-Schreyer [2009] we characterized the cohomology tables of vector bundles on $\mathbb{P}^{n}$ (up to a positive rational multiple) as the finite positive rational linear combinations of cohomology tables of supernatural bundles, which we described explicitly. In this paper we treat the cohomology tables of all coherent sheaves. These are given by infinite sums:

Theorem 0.1. The cohomology table of any coherent sheaf on $\mathbb{P}^{n}$ can be written as a convergent series, with positive real coefficients, of cohomology tables of supernatural bundles supported on linear subspaces.

We actually prove a more precise result, which includes a uniqueness statement. To state it we recall some ideas from Eisenbud-Schreyer [2009].

A sheaf $\mathcal{F}$ on $\mathbb{P}^{n}$ has supernatural cohomology if, for each integer $d$, the cohomology $H^{i}(\mathcal{F}(d))$ is nonzero for at most one value of $i$ and, in addition, the Hilbert polynomial $d \mapsto \chi(\mathcal{F}(d))$ has distinct integral roots. We define the root sequence of a supernatural sheaf $\mathcal{F}$ to be the sequence of roots of the Hilbert polynomial, written in decreasing order, $z_{1}>\cdots>z_{s}$ where $s$ is the dimension of the support of $\mathcal{F}$. It will be convenient to put $z_{0}=\infty$ and $z_{s+1}=z_{s+2}=\cdots=-\infty$.

D. Eisenbud: Department of Mathematics, University of California, Berkeley, Berkeley, CA 94720, USA; e-mail: de@msri.org

F.-O. Schreyer: Mathematik und Informatik, Universität des Saarlandes, Campus E2 4,

D-66123 Saarbrücken, Germany; e-mail: schreyer@math.uni-sb.de 
The Hilbert polynomial and the cohomology table of a supernatural sheaf $\mathcal{F}$ are determined by the root sequence $\left(z_{1}, \ldots, z_{s}\right)$ and the degree of $\mathcal{F}$ as follows. It is immediate that

$$
\chi(\mathcal{F}(d))=\frac{\operatorname{deg} \mathcal{F}}{s !} \prod_{i=1}^{s}\left(d-z_{i}\right) .
$$

By Theorem 6.4 of Eisenbud-Schreyer [2009],

$$
h^{j} \mathcal{F}(d)= \begin{cases}\frac{\operatorname{deg} \mathcal{F}}{s !} \prod_{i=1}^{s}\left|d-z_{i}\right| & \text { if } z_{j}>d>z_{j+1}, \\ 0 & \text { otherwise. }\end{cases}
$$

By Theorem 6.1 of that paper, there exists a supernatural sheaf of dimension $s$ and degree $s$ ! with any given root sequence $z=\left(z_{1}>\cdots>z_{s}\right)$. It may be taken to be a vector bundle on a linear subspace $\mathbb{P}^{s} \subset \mathbb{P}^{n}$. We denote its cohomology table by $\gamma^{z}$. Thus $\gamma^{z}$ is the cohomology table of a vector bundle on $\mathbb{P}^{n}$ if and only if $z_{n}>-\infty$.

We partially order the root sequences termwise, setting $z \geq z^{\prime}$ when $z_{1} \geq z_{1}^{\prime}, \ldots$, $z_{n} \geq z_{n}^{\prime}$. By a chain we mean a totally ordered set. If $Z$ is an infinite sequence of root sequences, $\left(q_{z}\right)_{z \in Z}$ a sequence of numbers, and $\gamma$ is a cohomology table, we write $\gamma=$ $\sum_{z \in Z} q_{z} \gamma^{z}$ to mean that each entry $\sum_{z \in Z} q_{z} \gamma_{i, d}^{z}$ converges to $\gamma_{i, d}$.

With these preparations we can state the precise version of our main result. Recall that a sheaf is said to be purely s-dimensional if all its associated subvarieties have dimension exactly $s$.

Theorem 0.2. Let $\gamma(\mathcal{F})$ be the cohomology table of a coherent sheaf $\mathcal{F}$ on $\mathbb{P}^{n}$. There is a chain of zero-sequences $Z$ and positive real numbers $q_{z}$ such that

$$
\gamma(\mathcal{F})=\sum_{z \in Z} q_{z} \gamma^{z}
$$

Both $Z$ and the numbers $q_{z}$ are uniquely determined by this condition. The coefficients $q_{z}$ corresponding to cohomology tables $\gamma^{z}$ of dimension $\operatorname{dim} \mathcal{F}$ are rational numbers. If $\mathcal{F}$ is purely $s$-dimensional, then all the $\gamma^{z}$ are cohomology tables of vector bundles on $\mathbb{P}^{s}$ and all the $q_{z}$ are rational.

Here are some open questions suggested by Theorem 0.2

1. Are all the numbers $q_{z}$ rational even when $\mathcal{F}$ is not pure-dimensional? Mats Boij (private communication) has proven that they are in the first special case beyond our theorem.

2. Any torsion-free sheaf $\mathcal{F}$ on $\mathbb{P}^{n}$ is purely $n$-dimensional, so all the $\gamma^{z}$ that appear in the decomposition of its cohomology table are cohomology tables of vector bundles. Is the converse true? 
When we want to display (parts) of a cohomology table we use the convention

$$
\begin{array}{ccccc|c}
\cdots & \gamma_{n,-n-1} & \gamma_{n,-n} & \gamma_{n,-n+1} & \cdots & n \\
& \vdots & \vdots & \vdots & & \vdots \\
\cdots & \gamma_{1,-2} & \gamma_{1,-1} & \gamma_{1,0} & \cdots & 1 \\
\cdots & \gamma_{0,-1} & \gamma_{0,0} & \gamma_{0,1} & \cdots & 0 \\
\hline \cdots & -1 & 0 & 1 & \cdots & d \backslash i
\end{array}
$$

We make this choice of indexing so that the cohomology table of a coherent sheaf $\mathcal{F}$ coincides with the Betti table of the Tate resolution of $\mathcal{F}$. This is a minimal, doubly infinite, exact free complex over the exterior algebra on $n+1$ generators that is associated to $\mathcal{F}$ by the Bernstein-Gel'fand-Gel'fand correspondence. It is studied in Eisenbud-FløystadSchreyer [2003] and Eisenbud-Schreyer [2003]. For consistency with the notation of those papers, we number the rows from the bottom and the columns from left to right as in the table above.

Example 0.3. The ideal sheaf $\mathcal{I}_{p}$ of a point in $\mathbb{P}^{2}$ has the cohomology table

$$
\begin{array}{ccccccccccc|c}
\cdots & 10 & 6 & 3 & 1 & & & & & & & 2 \\
\cdots & 1 & 1 & 1 & 1 & 1 & & & & & & 1 \\
& & & & & & 2 & 5 & 9 & 14 & \cdots & 0 \\
\hline \cdots & -4 & -3 & -2 & -1 & 0 & 1 & 2 & 3 & 4 & \cdots & d \backslash i
\end{array}
$$

where we drop the zero entries to make the shape visible. The expression guaranteed by Theorem 0.2 is

$$
\gamma\left(\mathcal{I}_{p}\right)=\sum_{k=2}^{\infty} q_{(0,-k)} \gamma^{(0,-k)}
$$

where

$$
q_{(0,-k)}=\frac{2}{(k-1) k(k+1)} .
$$

In particular, for $d \geq 1$,

$$
\sum_{k=2}^{\infty} \frac{2 d(d+k)}{(k-1) k(k+1)}=\left(\begin{array}{c}
d+2 \\
2
\end{array}\right)-1,
$$

while for $d \leq-1$,

$$
\sum_{k=-d+1}^{\infty} \frac{2 d(d+k)}{(k-1) k(k+1)}=-1
$$

and for $d \leq-2$,

$$
\sum_{k=2}^{-d} \frac{2 d(d+k)}{(k-1) k(k+1)}=\frac{(d+2)(d+1)}{2} .
$$


To explain the proof of Theorem 0.2 , we introduce a little more terminology. We define the $i$-th regularity of a table $\gamma \in \prod_{d=-\infty}^{\infty} \mathbb{R}^{n+1}$ to be

$$
z_{i}(\gamma)=\inf \left\{d \mid \gamma_{j, e+j}=0 \text { for all } j \geq i, e \geq d\right\} .
$$

We refer to $z(\gamma)=\left(z_{1}(\gamma), \ldots, z_{n}(\gamma)\right)$ as the regularity sequence of $\gamma$. It follows immediately from the definition that $z_{1}(\gamma)>z_{2}(\gamma)>\cdots$. Note that $z_{1}(\gamma(\mathcal{F}))$ coincides with the Castelnuovo-Mumford regularity of the sheaf $\mathcal{F}$. If $\gamma$ is the cohomology table of a supernatural sheaf $\mathcal{F}$, then it follows from Theorem 6.4 of Eisenbud-Schreyer [2009] that $z_{i}(\gamma)$ is the $i$-th root of the Hilbert polynomial of $\mathcal{F}$.

We define the support of a table $\gamma$ to be the set of indices $\left\{(i, d) \mid \gamma_{i, d} \neq 0\right\}$, and the dimension of $\gamma$ to be the maximum $i$ such that $\gamma_{i, d} \neq 0$ for some $d$, or -1 if all the $\gamma_{i, d}$ are zero. Finally, the corners and corner values of $\gamma$ are defined to be the positions $\left(i, z_{i}(\gamma)+i-1\right)$ and values $\gamma_{i, z_{i}}(\gamma)+i-1$ for each $i$ such that $i \leq \operatorname{dim} \gamma$ and $z_{i+1}<$ $z_{i}-1$. (See Example 0.5 and Figure 2 for pictures that may clarify this definition.) The decomposition of Theorem 0.2 is effected by a transfinite "greedy algorithm":

Algorithm 0.4 (Decompose a Cohomology Table).

Input: A cohomology table $\gamma=\gamma(\mathcal{F})$ for some coherent sheaf $\mathcal{F}$ on $\mathbb{P}^{n}$.

Output: A chain of root sequences $Z$ and positive real numbers $\left(q_{z}\right)_{z \in Z}$ such that $\gamma=$ $\sum_{z \in Z} q_{z} \gamma^{z}$.

1. Set $Z=\{\}$.

2. Set $i=\operatorname{dim} \gamma$.

3. WHILE $\operatorname{dim} \gamma=i$ DO

(a) Let $z$ be the regularity sequence of $\gamma$, and replace $Z$ by $Z \cup\{z\}$.

(b) Let $q_{z}>0$ be the largest real number such that the corner values of $\gamma$ are greater than or equal to the corner values of $q_{z} \gamma^{z}$.

(c) Replace $\gamma$ by $\gamma-q_{z} \gamma^{z}$.

4. Replace $\gamma$ by the limit of the tables produced in Step 3(c).

5. If $\gamma=0$ then STOP, else go to Step 2 .

Note that Step 2 is executed at most $n$ times, but we may loop through Steps 3(a)-3(c) infinitely often for each value of $i$ from $n$ to 1 .

Outline of the proof that Algorithm 0.4 succeeds. The crucial difficulty in the proof of Theorem 0.2 is to show that the table $\gamma-q_{z} \gamma^{z}$ produced each time we pass through Step 3(c) has nonnegative entries, and is sufficiently "like" the cohomology table of a coherent sheaf to allow us to continue. To do this we will define a class of tables closed under the basic operation in Step 3, and under taking limits in an appropriate way. We call these admissible tables; they are defined in $\$ 2$.

The proof that Step 3(c) produces an admissible table is also given in $\$ 2$. It rests on an understanding of some functionals that are positive on the cohomology tables of sheaves. Some of these functionals were defined in our paper [2009], and \$1 contains a simplified description of them, as well as some others necessary for the present proof. 
The dimension $s$ of $\gamma$ is genuinely reduced each time we return to Step 2: Indeed, some corner value of $\gamma$ becomes zero in Step 3(c), decreasing some $z_{i}$. Since $z_{s}$ remains the smallest of the (finite) $z_{i}$, only finitely many steps can occur before $z_{s}$ is reduced. Thus in the course of the WHILE loop $z_{s}$ must be reduced to $-\infty$, so the dimension drops in Step 4 if it has not dropped already in Step 3.

The convergence of the limiting process in Step 4 is dealt with in $\$ 3$ as are the uniqueness and the special case of a pure-dimensional sheaf. Finally, the necessary positivity is proven in $\$ 4$, following an idea suggested by Rob Lazarsfeld.

The following example shows that the decomposition of Theorem 0.2 sometimes mixes the torsion and torsion-free parts of a sheaf, even when the sheaf itself is a direct sum.

Example 0.5. Let $\mathcal{I}$ be the ideal sheaf of a point in $\mathbb{P}^{2}$, and let $L$ be a line in $\mathbb{P}^{2}$. Set $\mathcal{F}=\mathcal{I} \oplus \mathcal{O}_{L}(-4)$. The cohomology table of $\mathcal{F}$ is given by the following diagram, where we have marked the corner values with boxes.

\begin{tabular}{cccccccccc|c}
$\cdots$ & 6 & 3 & 1 & & & & & & & 2 \\
$\cdots$ & 8 & 7 & 6 & 5 & 3 & 2 & 1 & & & 1 \\
\cline { 5 - 7 } & & & & & 2 & 5 & 9 & 15 & $\cdots$ & 0 \\
\hline$\cdots$ & -3 & -2 & -1 & 0 & 1 & 2 & 3 & 4 & $\cdots$ & $d \backslash i$
\end{tabular}

The regularity sequence is $z=(-2,3)$. The supernatural cohomology table $\gamma^{z}$ is

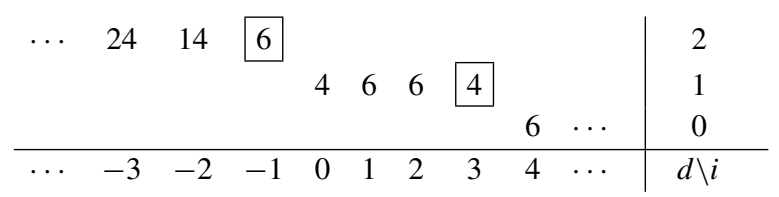

so we see that $q_{z}=1 / 6$. The table $\gamma^{\prime}:=\gamma-q_{z} \gamma^{z}$ has the form

$$
\begin{array}{cccccccccccc|c}
\cdots & 2 & 2 / 3 & & & & & & & & 2 \\
\cdots & 8 & 7 & 6 & 13 / 3 & 2 & 1 & 1 / 3 & & & 1 \\
& & & & & 2 & 5 & 9 & 14 & \cdots & 0 \\
\hline \cdots & -3 & -2 & -1 & 0 & 1 & 2 & 3 & 4 & \cdots & d \backslash i
\end{array}
$$

The regularity sequence of this table is $z^{\prime}=(-3,3)$. This time, the corner that is cancelled in $\gamma^{\prime}$ is the one in the middle row, which comes from the torsion sheaf $\mathcal{O}_{L}(-4)$, rather than from $\mathcal{I}$, and the table $\gamma^{\prime}-q_{z^{\prime}} \gamma^{z^{\prime}}$ looks like

\begin{tabular}{ccccccccccc|c}
$\cdots$ & $14 / 15$ & $1 / 5$ & & & & & & & & 2 \\
$\cdots$ & 8 & 7 & $17 / 3$ & $19 / 5$ & $7 / 5$ & $7 / 15$ & & & & 1 \\
& & & & & 2 & 5 & 9 & $203 / 15$ & $\cdots$ & 0 \\
\hline$\cdots$ & -3 & -2 & -1 & 0 & 1 & 2 & 3 & 4 & $\cdots$ & $d \backslash i$
\end{tabular}




\section{Positive functionals on cohomology tables}

In this section we will define some functionals - that is, real-valued functions- of an array

$$
\gamma=\left(\gamma_{j, d}\right) \in \prod_{d=-\infty}^{\infty} \mathbb{R}^{n+1} .
$$

The key to the proof of Theorem 0.2 is the Positivity Theorem 1.2 below, stating that certain of these functionals take nonnegative values on the cohomology tables of coherent sheaves.

Some of the functionals we need were defined in Eisenbud-Schreyer [2009], and Theorem 1.2 for those functionals, in the case of the cohomology table of a vector bundle, is a translation of what is there. Here we present a simpler account of the functionals, which adapts well to the new ones we use. The proof of Theorem 1.2 is given in $\$ 4$

Define the $t$-th partial Euler characteristic of the $d$-th twist of a table $\gamma \in$ $\prod_{d=-\infty}^{\infty} \mathbb{R}^{n+1}$ to be the functional

$$
\chi_{d}^{\leq t}(\gamma)=\sum_{i=0}^{t}(-1)^{i} \gamma_{i, d}
$$

When $t=\infty$ (or is simply large enough to be irrelevant) we simply write $\chi_{d}(\gamma)$ instead of $\chi_{d}^{\leq t}$. For example, the usual Euler characteristic of a sheaf $\mathcal{F}$ on $\mathbb{P}^{n}$ is $\chi(\mathcal{F})=$ $\chi_{0}^{\leq n}(\gamma(\mathcal{F}))=\chi_{0}(\gamma(\mathcal{F}))$.

If

$$
d:=d_{0}, \ldots, d_{s+1} \in \mathbb{Z}
$$

is a sequence of $s+2$ integers (which we will call degrees) and

$$
\psi=\psi_{0}, \ldots, \psi_{s+1}, \ldots \in \mathbb{Z} \cup\{\infty\}
$$

is a sequence of at least $s+1$ integers (which we will call bounds) then we set

$$
r_{i}=r_{i}(d):=\prod_{\substack{0 \leq j<k \leq s+1 \\ j, k \neq i}}\left(d_{k}-d_{j}\right)
$$

and define a functional $L(d, \psi): \prod_{d \in \mathbb{Z}} \mathbb{R}^{n+1} \rightarrow \mathbb{R}$ by the formula

$$
L(d, \psi)=\sum_{i=0}^{s+1}(-1)^{i} r_{i} \chi_{-d_{i}}^{\leq \psi_{i}}
$$

so that

$$
L(d, \psi)(\gamma)=\sum_{i=0}^{s+1}(-1)^{i} r_{i} \chi_{-d_{i}}^{\leq \psi_{i}}(\gamma)=\sum_{i=0}^{s+1}(-1)^{i} r_{i} \sum_{j=0}^{\psi_{i}}(-1)^{j} \gamma_{j,-d_{i}}
$$

We write $\infty$ for the special sequence of bounds $(\infty, \ldots, \infty, \ldots)$. The naturalness of the functionals $L(d, \psi)$ is suggested by the following well-known result from interpolation theory. 
Lemma 1.1. Let $d=\left(d_{0}, \ldots, d_{s+1}\right)$ be any sequence of $s+2$ numbers, and let $r_{i}=$ $r_{i}(d)$ as above. If $\gamma$ is the cohomology table of a coherent sheaf of dimension $\leq s$ (or any table of dimension $s$ such that $d \mapsto \chi_{d}(\gamma)$ is a polynomial of degree $\left.\leq s\right)$ then $L(d, \infty)(\gamma)=0$.

Proof. More generally, if $p(t)$ is any polynomial of degree $\leq s$ and $r_{i}=r_{i}(d)$, then

$$
\sum_{i=0}^{s+1}(-1)^{i} r_{i} p\left(d_{i}\right)=0 \text {. }
$$

This follows from the fact that the last column of the $(s+2) \times(s+2)$ matrix

$$
\left(\begin{array}{ccccc}
1 & d_{0} & \cdots & d_{0}^{s} & p\left(d_{0}\right) \\
1 & d_{1} & \cdots & d_{1}^{s} & p\left(d_{1}\right) \\
\vdots & \vdots & & \vdots & \vdots \\
1 & d_{s} & \cdots & d_{s}^{s} & p\left(d_{s}\right) \\
1 & d_{s+1} & \cdots & d_{s+1}^{s} & p\left(d_{s+1}\right)
\end{array}\right)
$$

is linearly dependent on the others, so the determinant vanishes. The displayed formula is the Laplace expansion of this determinant along the last column.

We will use the $L(d, \psi)$ with some other special sequences of bounds $\psi=\phi^{j}$ as well. For $j=1, \ldots, s$, we define

$$
\phi^{j}=\left(\phi_{0}^{j}, \ldots, \phi_{s+1}^{j}\right), \quad \text { where } \phi_{i}^{j}= \begin{cases}i & \text { if } i<j \\ i-1 & \text { if } i=j, \\ i-2 & \text { if } i>j\end{cases}
$$

or, less formally,

$$
\phi^{j}=(0, \ldots, j-2, j-1, j-1, j-1, j, \ldots, s-1) .
$$

Finally, we set

$$
\phi^{0}(s)=(-1,0, \ldots, s-2, s-1, s-1) .
$$

Here is our main result on the functionals $L\left(d, \phi^{j}(s)\right)$ :

Theorem 1.2 (Positivity). Let $d$ be a degree sequence, $d=\left(d_{0}<\cdots<d_{s+1}\right)$, and let $r=r(d)$. If $\mathcal{F}$ is a coherent sheaf on $\mathbb{P}^{n}$, then, for all $j \geq 1$

$$
L\left(d, \phi^{j}\right)(\gamma(\mathcal{F})) \geq 0 \text { and }-L\left(d, \phi^{0}\right)(\gamma(\mathcal{F})) \geq 0 .
$$

One may visualize the action of the linear form $L\left(d, \phi^{j}\right)$ on a cohomology table $\gamma$ as the dot product of $\gamma$ with the table illustrated (for the case $s=6, j=2$ ) in Figure 1 .

For the case $j>0$ the proof, given in $\$ 4$, follows the same outline as that in EisenbudSchreyer [2009]. Using the results of that paper and Boij-Söderberg [2008], we can see that Theorem 1.2, in the case $j>0$, is equivalent to Theorem 4.1. We will deduce the case $j=0$ from the case $j>0$ by a complicated numerical argument. It would be interesting to give a direct argument for the case $j=0$ as well.

Here is an example of how Theorem 1 can be applied. 


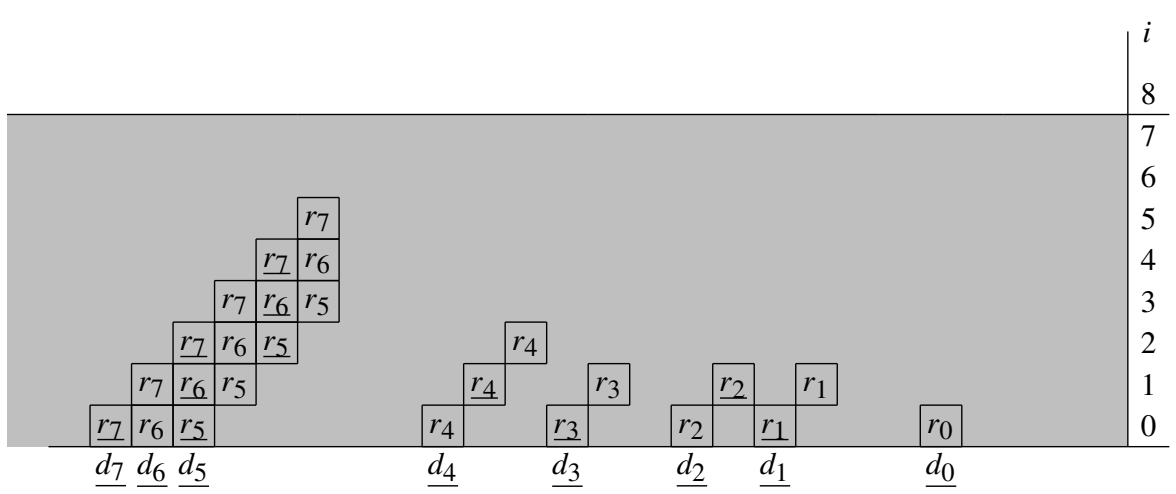

Fig. 1. To save space we have denoted $-d_{i}$ by $d_{i}$ and $-r_{i}$ by $r_{i}$. The shaded space indicates the positions where a cohomology table of a sheaf of dimension 7 on $\mathbb{P}^{8}$ could have nonzero values. The functional $L\left(d, \phi^{2}\right)$ is the dot product with the table having $\pm r_{i}$ in the positions shown, which are initial segments of the diagonals numbered $\underline{d_{0}}, \ldots, \underline{d_{7}}$, and zeros elsewhere.

Example 1.3. The Hilbert scheme $\operatorname{Hilb}^{2 t+2}\left(\mathbb{P}^{3}\right)=H_{1} \cup H_{2}$ has two irreducible components, which we will call $H_{1}$ and $H_{2}$. The generic point of $H_{1}$ corresponds to two skew lines $X \subset \mathbb{P}^{3}$, while the generic point of $H_{2}$ corresponds to $Y=C \cup p \subset \mathbb{P}^{3}$, where $C$ is a conic and $p$ is a point not in the plane spanned by $C$. The cohomology table of the ideal sheaf $\mathcal{I}_{X}$ is

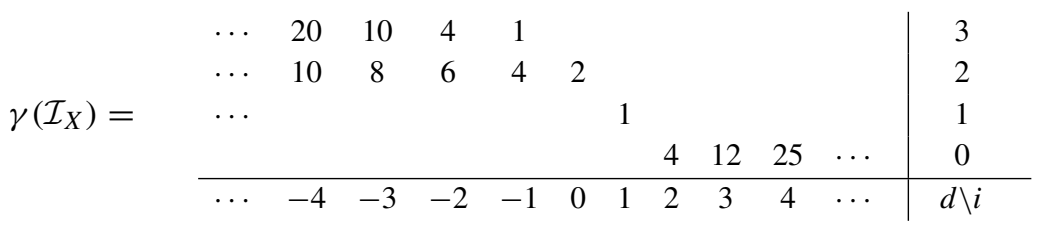

while that of $\mathcal{I}_{Y}$ is

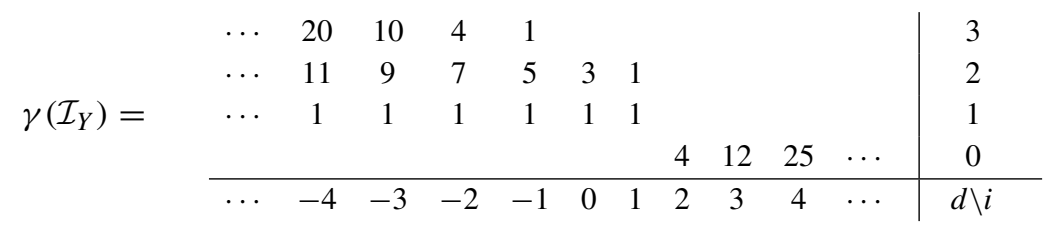

Using Theorem 1 we can show that any integral table "between" these two tables, obtained by replacing the value $h^{1} \mathcal{I}_{Y}(d)=1$ with a zero, and decreasing $h^{2} \mathcal{I}(d)$ by 1 as well, for some set of values $d<0$, cannot occur as the cohomology table of any sheaf; and even that no multiple of such a table can occur. For example, no multiple of either the table

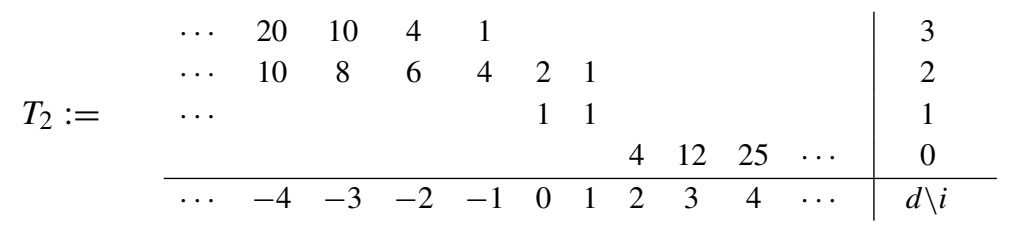


or

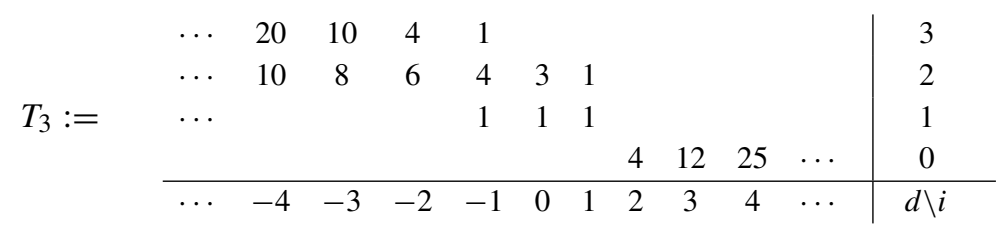

can be the cohomology table of a coherent sheaf.

One way to prove such a statement would be to apply Algorithm 0.4, and see that it eventually encounters a table with a negative entry. For instance, in the case of the table $T_{3}$, that occurs after 16 steps. But to prove the statement in general, it is easier to appeal directly to Theorem 1.2 .

First, consider the functional $L\left((-1,1,2,3), \phi^{2}\right)$, which may be written as the dot product with the table

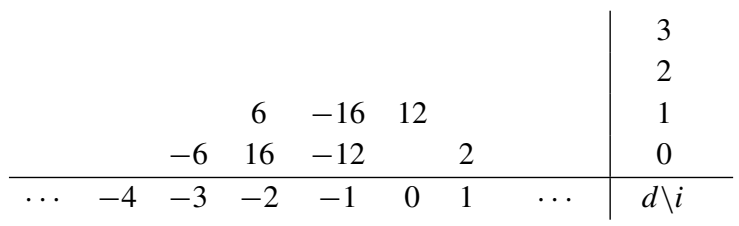

in which all entries not shown are zero. The value of this functional on the table $T_{3}$ shown above, for example, is $12-16=-4$, proving that no multiple of $T_{3}$ can be a cohomology table. Shifting this functional to $L\left((-1+e, 1+e, 2+e, 3+e), \phi^{2}\right)$ we get a collection of functionals that prove the corresponding statement for any table between $\gamma\left(\mathcal{I}_{X}\right)$ and $\gamma\left(\mathcal{I}_{Y}\right)$ that has the pattern 0,1 somewhere in the $h^{1}$ row, except for $T_{2}$. However, the functional $L\left((-1,0,1,2,5), \phi^{2}\right)$, which is given by the dot product with the table

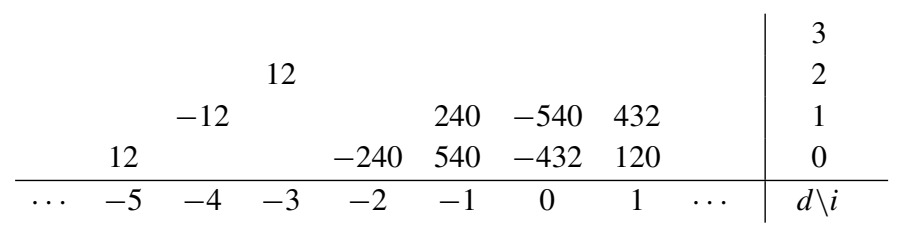

takes the value $432-540+12 \cdot 8=-12$ on $T_{2}$, proving the claim.

\section{Subtracting once}

As we execute Algorithm 0.4 we may leave the class of cohomology tables of coherent sheaves. We will say that a table is admissible if it satisfies conditions 1-3 below. As we shall see, the tables produced by the decomposition algorithm will all be admissible.

The first two conditions that an admissible table $\gamma \in \prod \mathbb{R}^{n+1}$ must satisfy are:

1. $\gamma_{i, d}=0$ for $i>0$ and $d \gg 0$.

2. The function $d \mapsto \chi_{d}(\gamma)$ from $\mathbb{Z}$ to $\mathbb{R}$ is a polynomial of degree $s^{\prime} \leq \operatorname{dim} \gamma$.

We will see that, in fact, admissibility implies that the degree of the polynomial in condition 2 is exactly $\operatorname{dim} \gamma$ (Corollary 2.2). 
For the last condition we need two definitions. Suppose that $\gamma$ is a table satisfying 1 and 2. Suppose that the dimension of $\gamma$ is $s$, and let $z_{1}>\cdots>z_{s}$ be the regularity sequence of $\gamma$, as defined above. We call the table positions

$$
\left\{(i, d) \mid z_{i+1}<d+i<z_{i}\right\}
$$

the top positions of $\gamma$, and all other positions with possibly nonzero values

$$
\left\{(i, d) \mid d+i \leq z_{i+1}\right\}
$$

the lower positions of $\gamma$. The last condition for admissibility is:

3. The values at the lower positions of $\gamma$ coincide with the values of the cohomology table of a coherent sheaf. That is, there exists a coherent sheaf $\mathcal{F}$ such that

$$
\gamma_{i, d}=h^{i}(\mathcal{F}(d)) \quad \text { for all lower positions }(i, d) \text { of } \gamma \text {. }
$$

Now let $\gamma$ be an admissible table of dimension $s$ with regularity sequence $z=z(\gamma)=$ $\left(z_{1}, \ldots, z_{s}\right)$, for example one whose shape is suggested by Figure 2

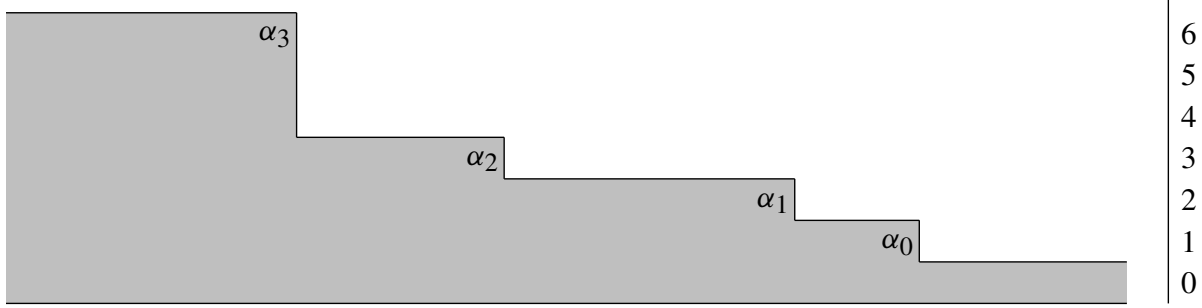

Fig. 2. A cohomology table of dimension $s=6$. The shaded region indicates where the table may have nonzero elements. The $\alpha_{i}$ are the corner values.

We want to subtract a suitable multiple $q_{z} \gamma^{z}$ of a supernatural table $\gamma^{z}$ so that, in $\gamma-q_{z} \gamma^{z}$, at least one of the corner values becomes zero, and the other corner values remain nonnegative. Figures 2 and 3 give an idea of the pattern.

$a_{3}$

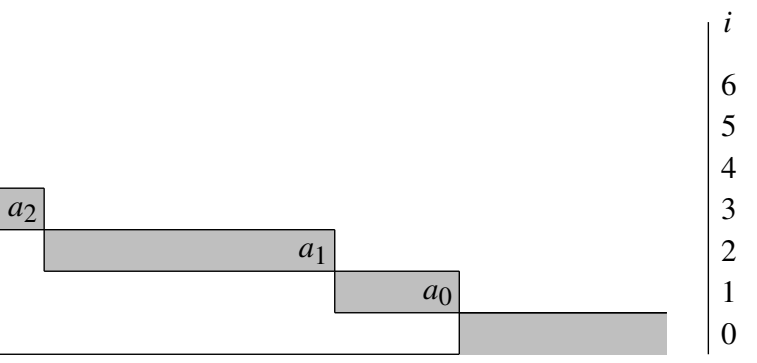

Fig. 3. Supernatural table $\gamma^{z}$ corresponding to the cohomology table in Figure 2 Here the $a_{i}$ are the corner values. The grayed area, where this table has nonzero values, coincides with the top positions of the table in Figure 2 
To achieve this goal we must take

$$
q_{z}=\min \left\{\alpha_{0} / a_{0}, \ldots, \alpha_{m} / a_{m}\right\},
$$

where $\alpha_{0}, \ldots, \alpha_{m}$ and $a_{0}, \ldots, a_{m}$ denote the corner values of $\gamma$ and $\gamma^{z}$ respectively. The main step in the proof of Theorem 0.2 is to show that all of the entries of $\gamma-q_{z} \gamma^{z}$ are nonnegative. This is the content of Proposition 2.1

Proposition 2.1. Let $\gamma$ be an admissible table of dimension $s>0$ with regularity sequence $z=\left(z_{1}, \ldots, z_{s}\right)$. Let $\gamma^{z}$ be the cohomology table of a supernatural sheaf of dimension $s=\operatorname{dim} \gamma$ with root sequence $z$. Let

$$
q_{z}=\min \left\{\frac{\gamma_{i, z_{i}+i-1}}{\gamma_{i, z_{i}+i-1}^{z}} \mid i \leq s \text { and } z_{i+1}<z_{i}-1\right\}
$$

be the minimal ratio of the corner values of $\gamma$ and $\gamma^{z}$. Then all entries of the table

$$
\gamma-q_{z} \gamma^{z}
$$

are nonnegative, and its regularity sequence is $<z$.

Corollary 2.2. If $\gamma$ is a nonzero admissible table, then the function $d \mapsto \chi_{d}(\gamma)$ is a polynomial of degree exactly $\operatorname{dim} \gamma$.

Proof of Corollary 2.2. For $d \gg 0$, the entry on the $d$-th diagonal of the table $\gamma^{z}$ is positive. Its value is $\prod_{i=1}^{s}\left(d-z_{i}\right)$, and thus grows as a polynomial of degree $s=\operatorname{dim} \gamma^{z}=$ $\operatorname{dim} \gamma$. If $d \mapsto \chi_{d}(\gamma)$ had degree $<\operatorname{dim} \gamma$, then $\gamma-q_{z} \gamma^{z}$ would have negative entries in these places, contradicting Proposition 2.1.

Proof of Proposition 2.1. Let $j$ be a cohomological index and $t$ a degree where $\gamma_{j, t}^{z} \neq 0$, say $z_{j+1}+j<t<z_{j}+j$. Let $\beta=\gamma_{j, d}$ and $b=\gamma_{j, d}^{z}$. We must show that $\beta-q_{z} b \geq 0$.

If $t=z_{j}+j-1$ then we are talking about values at a corner position of $\gamma$ and $\gamma^{z}$, and the assertion follows immediately from the definition of $q_{z}$. Thus we suppose that we are not at a corner position, that is, $z_{j+1}+j<t<z_{j}+j-1$.

We first treat the case where $j>0$. Figure 4 illustrates the situation for $j=2$.

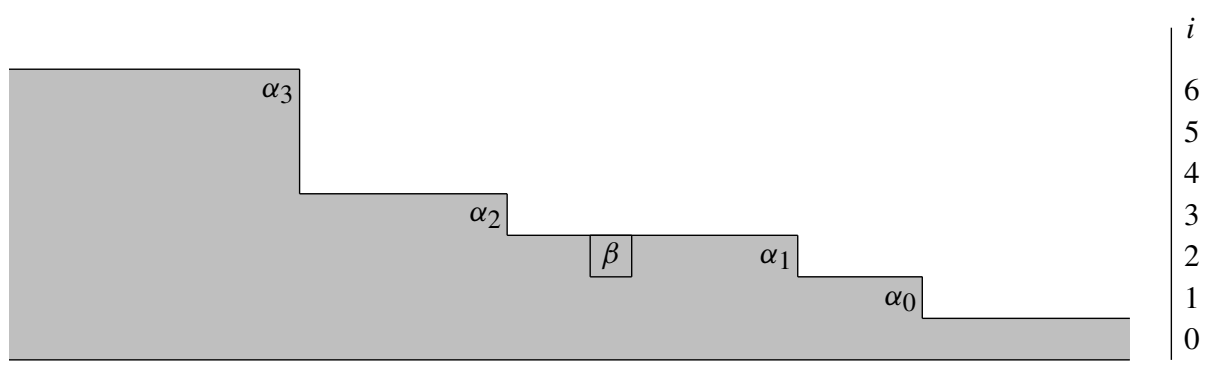

Fig. 4. The case $j>0$ (here $j=2$ ). We must prove that the entry $\beta-q_{z} b$, of the table $\gamma-q_{z} \gamma^{z}$, is nonnegative. Figure 5 shows the corresponding entry of $\gamma^{z}$. 


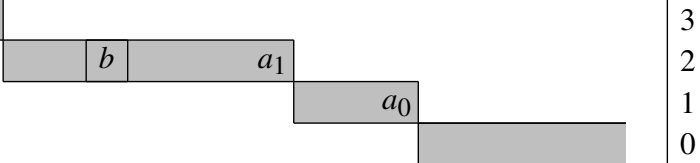

Fig. 5. Supernatural table $\gamma^{z}$ showing the value $b$ at the same position as that of $\beta$ in Figure 4

As indicated in the diagram, there is a corner position of $\gamma$ and $\gamma_{z}$ immediately to the right of the position $(j, t)$, and the values there are $\alpha_{i}:=\gamma_{j, z_{j}+j-1}$ and $a_{i}:=\gamma_{j, z_{j}+j-1}^{z}$ respectively. Since $\alpha_{i} / a_{i} \geq q_{z}$ it suffices to prove that

$$
\beta-\frac{\alpha_{i}}{a_{i}} b \geq 0
$$

To this end, consider the degree sequence

$$
d=\left(d_{0}, \ldots, d_{s+1}\right):=\left(-z_{1}, \ldots,-z_{j},-z_{j}+1,-t+j,-z_{j+1}, \ldots,-z_{s}\right)
$$

and let $r_{i}=r_{i}(d)$ as usual. Since $\chi_{z_{i}}\left(\gamma^{z}\right)=0$ by construction, Lemma 1.1 applied to the table $\gamma^{z}$ gives

$$
0=L(d, \infty)\left(\gamma^{z}\right)=\sum_{i=0}^{s+1}(-1)^{i} r_{i} \chi-d_{i}\left(\gamma^{z}\right)=r_{j} a_{i}-r_{j+1} b
$$

so $b / a_{i}=r_{j} / r_{j+1}$, and it suffices to show that $r_{j+1} \beta-r_{j} \alpha_{i} \geq 0$.

On the other hand, we may apply Lemma 1.1 to the admissible table $\gamma$ to get

$$
0=L(d, \infty)(\gamma)=\sum_{i=0}^{s+1}(-1)^{i} r_{i} \chi_{-d_{i}}(\gamma)=r_{j} \alpha_{i}-r_{j+1} \beta+L\left(d, \phi^{j}\right)(\gamma) .
$$

By the choice of the degree sequence $d$, the formula for $L\left(d, \phi^{j}\right)(\gamma)$ involves only values at the lower positions of $\gamma$ (see Figure 6).

Because $\gamma$ is admissible, $L\left(d, \phi^{j}\right)(\gamma)=L\left(d, \phi^{j}\right)(\gamma(\mathcal{F}))$ for some coherent sheaf $\mathcal{F}$. Thus we may apply Theorem 1.2 to conclude that

$$
r_{j+1} \beta-r_{j} \alpha_{i}=L\left(d, \phi^{j}\right)(\gamma) \geq 0
$$

as desired.

The proof in the case $j=0$ is almost the same. Figure 7 illustrates the position of the value $\beta$ in this case. Since $\gamma^{z}$ is assumed to be nonzero at the position $(j, t)$, we must have $t>z_{1}$ in this case. This time there is no corner position to the right of $(0, t)$, but we set $i=m$, and we let $d$ be the degree sequence

$$
d=\left(d_{0}, \ldots, d_{s+1}\right)=\left(-t,-z_{1}, \ldots,-z_{s},-z_{s}+1\right) .
$$




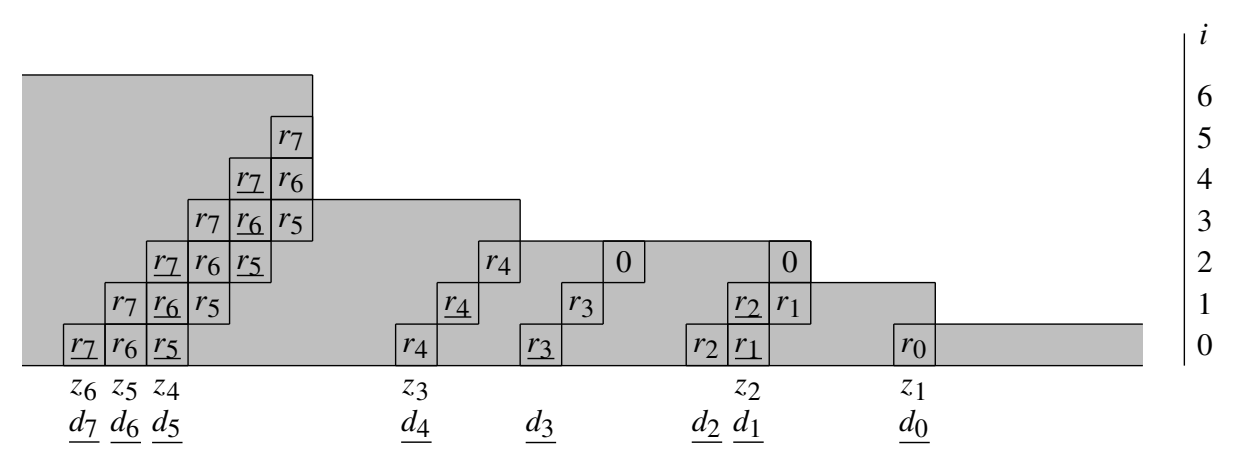

Fig. 6. The functional $L\left(d, \phi^{j}\right)$ is the dot product with the table having $\pm r_{i}$ in the positions shown, and zeros elsewhere. In the illustration, $s=6$ and $j=2$. To save space we have denoted $-d_{i}$ by $\underline{d_{i}}$ and $-r_{i}$ by $\underline{r}_{i}$. The explicit zeros are added for emphasis.

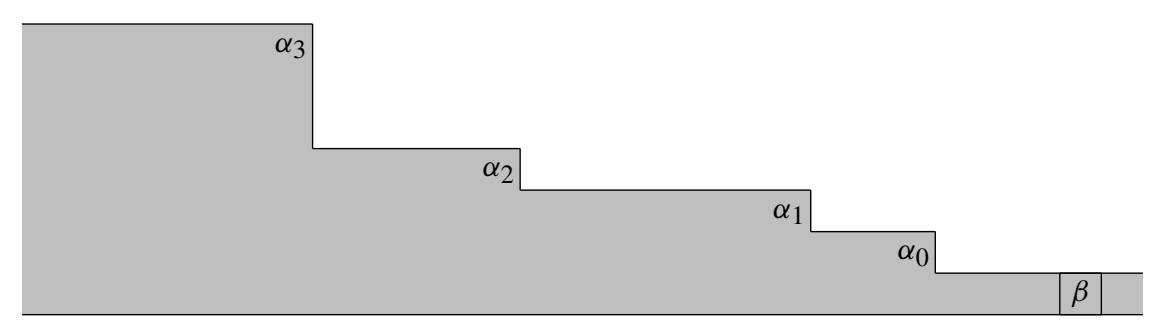

Fig. 7. Position of $\beta$ in case $j=0$.

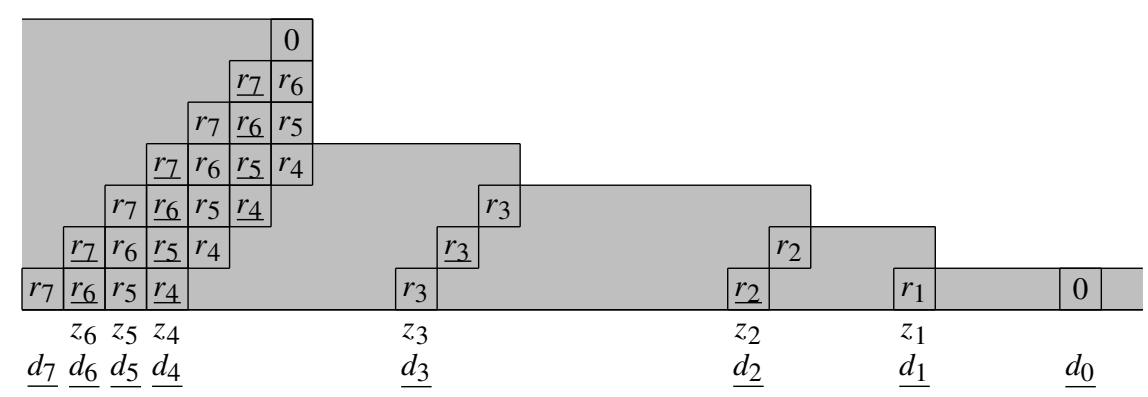

Fig. 8. The functional $-L\left(d, \phi^{0}\right)$ is the dot product with the table having $\pm r_{i}$ in the positions shown, and zeros elsewhere. In the illustration, $s=6$. To save space we have denoted $-d_{i}$ by $\underline{d_{i}}$ and $-r_{i}$ by $r_{i}$. The explicit zeros are added for emphasis.

Figure 8 illustrates the relation of $\gamma$ to the positions involved in the functional $-L\left(d, \phi^{0}\right)$. The rest of the argument is nearly the same:

$$
\beta-q_{z} b \geq \beta-\frac{\alpha_{m}}{a_{m}} b \geq 0
$$

follows, because

$$
0=L(d, \infty)\left(\gamma^{z}\right)=r_{0} b-r_{s+1} a_{m}
$$


gives $b / a_{m}=r_{s+1} / r_{0}$, and

$$
0=L(d, \infty)(\gamma)=r_{0} \beta-r_{s+1} \alpha_{m}+L\left(d, \phi^{0}\right)(\gamma)
$$

implies the desired positivity, because $-L\left(d, \phi^{0}\right)(\gamma) \geq 0$ by Theorem 1

\section{Proof of the main result}

We start by describing the growth of dimensions of the cohomology groups $h^{i}(\mathcal{F}(d))$ for $d \gg 0$.

Proposition 3.1. Let $\mathcal{F}$ be a coherent sheaf on $\mathbb{P}^{n}$. For each $i=0, \ldots, n$ there exists a polynomial $p_{\mathcal{F}}^{i} \in \mathbb{Q}[t]$ such that

$$
p_{\mathcal{F}}^{i}(d)=h^{i}(\mathcal{F}(d)) \quad \text { for all } d \ll 0 .
$$

The degree of $p_{\mathcal{F}}^{i}$ is $\leq i$, with equality if and only if $\mathcal{F}$ has an associated subvariety of dimension $i$. In particular, if the dimension of the support of $\mathcal{F}$ is $s$, then $\operatorname{deg} p_{\mathcal{F}}^{s}=s$. Furthermore, $\mathcal{F}$ is pure-dimensional if and only if $\operatorname{deg} p_{\mathcal{F}}^{i}<i$ for every $i<s$.

Proof. Let $M$ be a graded module over the polynomial ring $S=\mathbb{K}\left[x_{0}, \ldots, x_{n}\right]$ whose associated sheaf is $\mathcal{F}$. For $i>0$,

$$
\begin{aligned}
\bigoplus_{d} \operatorname{Hom}_{\mathbb{K}}\left(H^{i}(\mathcal{F}(d)), \mathbb{K}\right) & =\bigoplus_{d} \operatorname{Ext}^{n-i}\left(\mathcal{F}(d), \omega_{\mathbb{P} n}\right) \\
& =\bigoplus_{d} \operatorname{Ext}^{n-i}(\mathcal{F}, \mathcal{O}(-n-1-d)) \\
& =\operatorname{Ext}_{S}^{n-i}(M, S(-n-1)) .
\end{aligned}
$$

Thus $p_{\mathcal{F}}^{i}$ is the Hilbert polynomial of $\operatorname{Ext}_{S}^{n-i}(M, S(-n-1))$, so the degree of $p_{\mathcal{F}}^{i}$ is one less than the Krull dimension of $\operatorname{Ext}_{S}^{n-i}(M, S(-n-1))$, or, equivalently, of $\operatorname{Ext}_{S}^{n-i}(M, S)$.

The inequality $\operatorname{deg} p_{\mathcal{F}}^{i} \leq i$ now follows from the Auslander-Buchsbaum-Serre Theorem: after localizing $S$ at any prime $P$ of dimension $>i+1$ we get a regular local ring of dimension $<(n+1)-(i+1)=n-i$, so $\operatorname{Ext}^{n-i}(M, S)_{P}=0$. It follows that $\operatorname{dim} \operatorname{Ext}^{n-i}(M, S)<i+1$. Now suppose that $P$ is a prime of dimension exactly $i+1$. By the Auslander-Buchsbaum formula, $P$ is associated to $M$ if and only if the projective dimension of $M_{P}$ is $i+1$, which is true if and only if $\operatorname{Ext}^{n-i}(M, S)_{P} \neq 0$. Since every associated prime of a graded module is homogeneous, $P$ must correspond in this case to an associated subvariety of $\mathcal{F}$, proving the statement about equality. The rest of the proposition follows.

Proof of Theorem 0.2 For the first statement of the Theorem it suffices to show that Algorithm 0.4 succeeds. We have already seen in Proposition 2.1 that Step 3(c), starting with an admissible table, always produces a new admissible table, and we have explained in the Outline of the Proof in the Introduction why the dimension of $\gamma$ will drop by at least 1 each time we reach Step 2. Thus it suffices to show that if we start with an 
admissible table $\gamma$, then the sequence of tables produced by the WHILE loop of Step 3 actually converges to an admissible table, so that we can execute Step 4.

Convergence is no problem: By Proposition 2.1, the tables stay admissible, and thus have only nonnegative terms throughout an instance of Step 3. Thus the values in a given position form a decreasing, bounded below sequence.

To show that the limiting table produced in Step 4 is admissible, suppose the rows of cohomological index $s^{\prime}+1, \ldots, s$ are wiped out by a pass through Step 3, while the $s^{\prime}$-th row remains nonzero. We have to show that the remaining table $\gamma^{\prime}$ is an admissible table of dimension $s^{\prime}$. It is clear, in any case, that $\gamma^{\prime}$ satisfies condition 1 of admissibility.

Since the rows with cohomological index $0, \ldots, s^{\prime}$ survive, only finitely many corner values with cohomological index $j \leq s^{\prime}$ are removed in the course of Step 3. Thus we may replace $\gamma$ with the admissible table that results from finitely many subtractions, and assume that no corner value with cohomological index $\leq s^{\prime}$ becomes zero in the infinite sequence of subtractions leading to $\gamma^{\prime}$. It follows that the values of in the lower positions of $\gamma^{\prime}$ are the same as those in the corresponding positions of $\gamma$; thus condition 3 of admissibility is satisfied.

To complete the proof, we first note that the sequence of Hilbert functions of the tables obtained by the successive subtractions converges decreasingly to a function that takes nonnegative real values at all $d \gg 0$. At every finite stage we subtract a polynomial of degree $s+1$, so the $s+1$-st difference function is zero. By continuity, it remains zero in the limit. It follows that $d \mapsto \chi_{d}\left(\gamma^{\prime}\right)$ is a polynomial function.

On the other hand, the values on the top row of $\gamma^{\prime}$ at the positions $d \ll 0$ grow at most like a polynomial of degree $s^{\prime}$ since all values are bounded by the values of the corresponding row of $\gamma$. The rows with cohomological degree $i<s^{\prime}$ have for $d \ll 0$ the values of the original cohomology table of $\mathcal{F}$. By Proposition 3.1, they grow with negative $d$ as polynomials of degree $<s^{\prime}$. Thus the Euler characteristic $\chi_{d}(\gamma)$ is a polynomial in $d$ of degree $\leq s^{\prime}$; that is, $\gamma^{\prime}$ satisfies condition 2 of admissibility. This completes the proof that Algorithm 0.4 succeeds, and produces a decomposition of the desired kind.

To prove uniqueness, suppose that $Z$ and $W$ are both chains of root sequences, and that

$$
\gamma(\mathcal{F})=\sum_{z \in Z} q_{z} \gamma^{z}=\sum_{w \in W} r_{w} \gamma^{w}
$$

with $q_{z}$ and $r_{w}$ positive real numbers, where $Z$ is the chain produced by Algorithm 0.4 Since $Z$, at least, is well ordered, there is a largest element of $Z$ that does not appear in $W$, or appears with a different coefficient. We may as well subtract the contributions of the terms corresponding to larger elements of $Z$, which are the same for the two sums, and thus suppose that

$$
\gamma=\sum_{z \in Z} q_{z} \gamma^{z}=\sum_{w \in W} r_{w} \gamma^{w}
$$

where $\gamma$ is an admissible table, and the maximal element $\bar{z} \in Z$ either does not appear in $W$, or appears with a different coefficient $r_{\bar{z}} \neq q_{\bar{z}}$.

Because the root sequence of $Z$ is the regularity sequence of $\gamma$, every $w \in W$ must satisfy $w \leq \bar{z}$. If $\bar{z}$ itself is in $W$, but $r_{\bar{z}} \neq q_{\bar{z}}$, then $\gamma-r_{\bar{z}} \gamma^{\bar{z}}$ has exactly the same corner 
positions and regularity sequence as $\gamma$. But since $W$ is a chain, at least one of the corner positions of $\gamma$ is represented with the value zero in every one of the $\gamma^{w}$ for $\bar{z} \neq w \in W$, and we see that $\gamma-\sum_{w \in W} r_{w} \gamma^{w} \neq 0$, contradicting our hypothesis.

Similarly, if $\bar{z} \notin W$ then, since there are only finitely many elements just below $z$ in the poset of root sequences, there is some corner position of $\gamma$ that is represented by the value zero in every $\gamma^{w}$ for $w \in W$, so we can finish the argument in the same way. This proves uniqueness.

Note that the coefficients $q_{z}$ involved in any finite sequence of subtractions in Algorithm 0.4 starting from a rational cohomology table are automatically rational. This applies to all the $q_{z}$ corresponding to $\gamma^{z}$ of dimension $=\operatorname{dim} \mathcal{F}$.

Now suppose that $\mathcal{F}$ is a pure-dimensional sheaf. It suffices to show that Algorithm 0.4 terminates the first time that Step 5 is reached, since before taking the limit in Step 4, all coefficients needed are rational.

Once again, let $\gamma^{\prime}$ be the result of subtracting the cohomology tables of vector bundles on $\mathbb{P}^{s}$, as in Algorithm 0.4 , so that $s^{\prime}:=\operatorname{dim} \gamma^{\prime}<\operatorname{dim} \gamma(\mathcal{F})=s$. By Proposition 3.1, the Euler characteristic of the resulting table $\gamma^{\prime}$ grows like a polynomial of degree $\leq s^{\prime}-1$. If $\gamma^{\prime}$ were nonzero, we would get a contradiction to Corollary 2.2. Thus $\gamma^{\prime}=0$, completing the proof.

\section{Proof of the positivity theorem}

In Eisenbud-Schreyer [2009] we defined pairings

$$
\langle\beta, \gamma\rangle=\sum_{\{i, j, k \mid j \leq i\}}(-1)^{i-j} \beta_{i, k} \gamma_{j,-k}
$$

and

$$
\begin{aligned}
\langle\beta, \gamma\rangle_{c, \tau}= & \sum_{\{i, j, k \mid j \leq i \text { and }(j<\tau \text { or } j \leq i-2)\}}(-1)^{i-j} \beta_{i, k} \gamma_{j,-k} \\
& +\sum_{\{i, j, k, \epsilon \mid 0 \leq \epsilon \leq 1, j=\tau, i=j+\epsilon, k \leq c+\epsilon\}}(-1)^{i-j} \beta_{i, k} \gamma_{j,-k}
\end{aligned}
$$

for $\beta=\left(\beta_{i, k}\right) \in \bigoplus_{-\infty}^{\infty} \mathbb{R}^{n+2}$ and $\gamma \in \prod_{-\infty}^{\infty} \mathbb{R}^{n+2}$, and $0 \leq \tau \leq n, c \in \mathbb{Z}$. We showed that if $\beta$ is the Betti table of a finitely generated graded module over $S:=\mathbb{K}\left[x_{0}, \ldots, x_{n}\right]$ and $\gamma$ is the cohomology table of a vector bundle $\mathcal{F}$, or of a complex $E$ of free graded $S$-modules, supported in positive cohomological degrees, then

$$
\langle\beta, \gamma\rangle \geq 0 \text { and }\langle\beta, \gamma\rangle_{c, \tau} \geq 0 .
$$

Our proof for the vector bundle case reduced to the case of a free complex by replacing the vector bundle with a free monad. Since the free monads of coherent sheaves have terms in negative cohomological degrees, this proof could not show that the pairing above was nonnegative when $\mathcal{F}$ is a general coherent sheaf. After our paper was finished, Rob Lazarsfeld pointed out to us a variation on our proof in which the monad for $\mathcal{F}$ is replaced by an injective or flasque resolution of $\mathcal{F}$. It turns out that, with one further idea, this idea yields a proof of nonnegativity that works for any coherent sheaf $\mathcal{F}$. 
Theorem 4.1. Let $F$ be the minimal free resolution of a finitely generated graded $S$ module $M$. If $\mathcal{F}$ is a coherent sheaf on $\mathbb{P}^{n}$, then

$$
\langle F, \mathcal{F}\rangle \geq 0 \quad \text { and } \quad\langle F, \mathcal{F}\rangle_{c, \tau} \geq 0 .
$$

Proof. The number $\langle F, \mathcal{F}\rangle$ depends only on the dimensions of the $H^{j}(\mathcal{F}(-k))$ for $k \in \mathbb{Z}$, we may begin by replacing $\mathcal{F}$ with a "general translate" by an element of $P G L(n)$, to make $\mathcal{F}$ homologically transverse to the sheaf $\tilde{M}$, as proven by Sierra [2007] and by Miller and Speyer [2008]. If we let $G$ be a graded $S$-module such that $\widetilde{G}=\mathcal{F}$, this means that the modules $\operatorname{Tor}_{i}^{S}(M, G)$ have support only at the irrelevant ideal for $i>0$.

Let $E: \bigoplus_{\ell} G\left[x_{\ell}^{-1}\right] \rightarrow \cdots$ be the Čech complex of $G$. The homological transversality implies that the complex $F \otimes E^{j}$ has homology only at $F_{0} \otimes E^{j}$, so the total complex of the double complex $F \otimes E$ has homology only in nonnegative cohomological degree. We can now proceed exactly as in the proofs of Theorems 3.1 and 4.1 of Eisenbud-Schreyer [2009].

We next describe a simplification in the statement of Theorem 4.1 that makes use of the main results of Eisenbud-Schreyer [2009] and of Boij-Söderberg [2008], and also an extension of the statement that will be crucial for the proof of Theorem 0.2 .

Recall that a graded Cohen-Macaulay $S$-module $M$ of codimension $s+1$ is said to have a pure resolution with degree sequence $d=\left(d_{0}, \ldots, d_{s+1}\right)$ if the minimal free resolution of $M$ has the form

$$
S\left(-d_{0}\right)^{r_{0}} \leftarrow S\left(-d_{1}\right)^{r_{1}} \leftarrow \cdots \leftarrow S\left(-d_{s+1}\right)^{r_{s+1}} \leftarrow 0 .
$$

In this case, $d_{0}<\cdots<d_{s+1}$, and there is a positive rational number $q$ such that each $r_{i}$ equals $q \cdot r_{i}(d)$, where, as in $\$ 4$.

$$
r_{i}(d):=\prod_{\substack{1 \leq j<k \leq s+1 \\ j, k \neq i}}\left(d_{k}-d_{j}\right)
$$

(see Herzog and Kühl [1984]).

Together, Eisenbud-Schreyer [2009] and Boij-Söderberg [2008] show that there is a graded Cohen-Macaulay $S$-module with any given degree sequence $\left(d_{0}<\cdots<d_{s+1}\right)$, and the Betti table of any graded $S$-module is a positive rational linear combination of the Betti tables of Cohen-Macaulay modules with pure resolutions. Thus to prove that the value of a bilinear functional such as those above is nonnegative, it suffices to treat the case where $\beta$ is the Betti table of a Cohen-Macaulay module with pure resolution, and if the resolution has degree sequence $d$, one may as well assume that $r_{i}=r_{i}(d)$ for every $i$ as well: that is, we may restrict our attention to the functionals $\left\langle\left(\beta^{d}, \gamma\right\rangle_{c, \tau}\right.$ with $\beta^{d}$ to be the table with

$$
\beta^{d}: \quad \beta_{i, j}= \begin{cases}r_{i}(d) & \text { if } j=d_{i} \\ 0 & \text { otherwise }\end{cases}
$$


For such $\beta^{d}$ we may rewrite the definition given above in the form

$$
\begin{aligned}
\left\langle\beta^{d}, \gamma\right\rangle_{c, \tau}= & \sum_{i<\tau}(-1)^{i} r_{i}(d) \chi_{-d_{i}}^{\leq i}(\gamma)+(-1)^{\tau} r_{\tau}(d) \chi_{-d_{\tau}}^{\leq A}(\gamma) \\
& +(-1)^{\tau+1} r_{\tau+1}(d) \chi_{-d_{\tau+1}}^{\leq B}(\gamma)+\sum_{i>\tau+1}(-1)^{i} r_{i}(d) \chi_{-d_{i}}^{\leq i-2}(\gamma)
\end{aligned}
$$

where

$$
A=\left\{\begin{array}{ll}
\tau-1 & \text { if } c<d_{\tau}, \\
\tau & \text { otherwise, }
\end{array} \quad B= \begin{cases}\tau-1 & \text { if } c<d_{\tau+1}, \\
\tau & \text { otherwise, }\end{cases}\right.
$$

It follows that if $\tau \geq 1$ and $c<d_{\tau}$ then

$$
\left\langle\beta^{d}, \gamma\right\rangle_{c, \tau}=L\left(d, \phi^{\tau}\right)(\gamma)
$$

while if $c \geq d_{\tau+1}$ then

$$
\left\langle\beta^{d}, \gamma\right\rangle_{c, \tau}=L\left(d, \phi^{\tau+1}\right)(\gamma) .
$$

Moreover, if the $\gamma_{i, j}$ are nonnegative, as in any admissible table, and $d_{\tau} \leq c<d_{\tau+1}$, then, comparing signs, we see that

$$
\left\langle\beta^{d}, \gamma\right\rangle_{c, \tau} \geq\left\langle\beta^{d}, \gamma\right\rangle_{d_{\tau}-1, \tau}=L\left(d, \phi^{\tau}\right)(\gamma),
$$

so this case is not very useful.

Proof of Theorem 1.2. The description above shows that the cases $j>0$ follow from Theorem 4.1 .

To simplify the notation for the case $j=0$ we set $\psi=\phi^{0}=(-1,0,1, \ldots, s-2$, $s-1, s-1)$. We write

$$
d^{(j)}=\left(d_{1}, \ldots, d_{j}\right) \quad \text { for } j=1, \ldots, s+1
$$

and

$$
\psi^{(j)}= \begin{cases}(0,1, \ldots, j-1) & \text { for } j=1, \ldots, s \\ (0,1, \ldots, s-1, s-1) & \text { for } j=s+1\end{cases}
$$

We will show that

$$
-L(d, \psi)=\sum_{\ell=0}^{s+1}(-1)^{s-\ell} r_{s+1-\ell}(d) \chi_{-d_{s+1-\ell}}^{\leq \psi_{s+1-\ell}}=\sum_{k=0}^{s} A_{k} L\left(d^{(s+1-k)}, \psi^{(s+1-k)}\right)
$$

where

$$
A_{k}=\prod_{1 \leq j \leq s-k}\left(d_{j}-d_{0}\right) \prod_{\substack{1 \leq i<j \leq s+1 \\ s+1-k<j}}\left(d_{j}-d_{i}\right)
$$

The coefficients $A_{k}$ are obviously nonnegative. By Theorem 4.1, the forms $L\left(d^{(s+1-k)}, \psi^{(s+1-k)}\right)$ take nonnegative values on the cohomology tables of coherent sheaves, so this will suffice to prove Theorem 1.2 . 
The coefficient of $(-1)^{s-\ell} \chi_{-d_{s+1-\ell}}^{\leq \psi_{s+1-\ell}}$ on the right-hand side of (1) is

$$
\sum_{k=0}^{\ell}\left(\prod_{1 \leq j \leq s-k}\left(d_{j}-d_{0}\right)\right)\left(\prod_{\substack{1 \leq i<j \leq s+1 \\ s+1-k<j}}\left(d_{j}-d_{i}\right)\right)\left(\prod_{\substack{1 \leq i<j \leq s+1-k \\ i, j \neq s+1-\ell}}\left(d_{j}-d_{i}\right)\right) .
$$

We will show that this is $r_{s+1-\ell}(d)$. The terms in the sum have a common factor (coming from the first and third factors in each term)

$$
\left(\prod_{1 \leq j \leq s-\ell}\left(d_{j}-d_{0}\right)\right)\left(\prod_{\substack{1 \leq i<j \leq s+1-\ell \\ i, j \neq s+1-\ell}}\left(d_{j}-d_{i}\right)\right)=\prod_{\substack{0 \leq i<j \leq s+1-\ell \\ i, j \neq s+1-\ell}}\left(d_{j}-d_{i}\right) .
$$

After factoring this out, we get

$$
\sum_{k=0}^{\ell}\left(\prod_{s-\ell+1 \leq j \leq s-k}\left(d_{j}-d_{0}\right)\right)\left(\prod_{\substack{1 \leq i<j \leq s+1 \\ s+1-k<j}}\left(d_{j}-d_{i}\right)\right)\left(\prod_{\substack{1 \leq i<j \leq s+1 \\ i, j \neq s+1-\ell \\ s+1-\ell<j \leq s+1-k}}\left(d_{j}-d_{i}\right)\right)
$$

which can be further factored as

$$
\left(\prod_{\substack{1 \leq i<j \leq s+1 \\ i, j \neq s+1-\ell \\ s+1-\ell<j}}\left(d_{j}-d_{i}\right)\right) \sum_{k=0}^{\ell}\left(\prod_{s-\ell+1 \leq j \leq s-k}\left(d_{j}-d_{0}\right)\right)\left(\prod_{\substack{i=s+1-\ell \\ s-k+1<j}}\left(d_{j}-d_{i}\right)\right) .
$$

Applying the case $t=-1$ of Lemma 4.2. we can combine all the factors to express the original sum as

$$
\begin{gathered}
\left(\prod_{\substack{0 \leq i<j \leq s+1-\ell \\
i, j \neq s+1-\ell}}\left(d_{j}-d_{i}\right)\right)\left(\prod_{\substack{1 \leq i<j \leq s+1 \\
i, j \neq s+1-\ell \\
s+1-\ell<j}}\left(d_{j}-d_{i}\right)\right)\left(\prod_{s-\ell+1<j \leq s+1}\left(d_{j}-d_{0}\right)\right) \\
=\prod_{\substack{0 \leq i<j \leq s+1 \\
i, j \neq s+1-\ell}}\left(d_{j}-d_{i}\right)=r_{s+1-\ell}(d),
\end{gathered}
$$

completing the proof.

Lemma 4.2. For $-1 \leq t \leq \ell-1$ we have

$$
\begin{aligned}
\sum_{k=0}^{\ell}\left(\prod_{s-\ell+1 \leq j \leq s-k}\left(d_{j}-d_{0}\right)\right)\left(\prod_{s-k+1<j}\left(d_{j}-d_{s-\ell+1}\right)\right) & \left(\prod_{s-\ell+1<j \leq s-t}\left(d_{j}-d_{0}\right)\right)\left(\prod_{s-t<j}\left(d_{j}-d_{s-\ell+1}\right)\right) \\
& +\sum_{k=0}^{t}\left(\prod_{s-\ell+1 \leq j \leq s-k}\left(d_{j}-d_{0}\right)\right)\left(\prod_{s-k+1<j}\left(d_{j}-d_{s-\ell+1}\right)\right)
\end{aligned}
$$


Proof. The formula is obvious for $t=\ell-1$, so we do descending induction. The induction step follows by combining the first product with the $k=t$ term of the summation, as follows:

$$
\begin{gathered}
\prod_{j=s-\ell+2}^{s-t}\left(d_{j}-d_{0}\right) \prod_{s-t<j}\left(d_{j}-d_{s-\ell+1}\right)+\prod_{j=s-\ell+1}^{s-t}\left(d_{j}-d_{0}\right) \prod_{s-t+1<j}\left(d_{j}-d_{s-\ell+1}\right) \\
=\left(\prod_{j=s-\ell+2}^{s-t}\left(d_{j}-d_{0}\right)\right)\left(\left(d_{s-t+1}-d_{s-\ell+1}\right)+\left(d_{s-\ell+1}-d_{0}\right)\right)\left(\prod_{s-(t-1)<j}\left(d_{j}-d_{s-\ell+1}\right)\right) \\
=\left(\prod_{j=s-\ell+2}^{s-(t-1)}\left(d_{j}-d_{0}\right)\right)\left(\prod_{s-(t-1)<j}\left(d_{j}-d_{s-\ell+1}\right)\right) .
\end{gathered}
$$

\section{References}

Boij, M., Söderberg, J. [2006]: Graded Betti numbers of Cohen-Macaulay modules and the multiplicity conjecture. J. London Math. Soc. 78, 85-106 (2008) Zbl pre05309689 MR 2427053

Boij, M., Söderberg, J. [2008]: Betti numbers of graded modules and the multiplicity conjecture in the non-Cohen-Macaulay case. arXiv:0803.1645.

Eisenbud, D., Fløystad, G., Schreyer, F.-O. [2003]: Sheaf cohomology and free resolutions over exterior algebras. Trans. Amer. Math. Soc. 355, 4397-4426 (2003) Zbl 1063.14021 MR 1990756

Eisenbud, D., Fløystad, G., Weyman, J. [2007]: The existence of pure free resolutions. arXiv:0709.1529.

Eisenbud, D., Schreyer, F.-O. [2003]: Resultants and Chow forms via exterior syzygies. J. Amer. Math. Soc. 16, 537-579 (2003) Zbl 1069.14019 MR 1969204

Eisenbud, D., Schreyer, F.-O. [2009]: Betti numbers of graded modules and cohomology of vector bundles, J. Amer. Math. Soc. 22, 859-888 (2009) MR 2505303

Herzog, J., Kühl, M. [1984]: On the Betti numbers of finite pure and linear resolutions. Comm. Algebra 12, 1627-1646 (1984) Zbl 0543.13008 MR 0743307

Miller, E., Speyer, D. [2008]: A Kleiman-Bertini theorem for sheaf tensor products. J. Algebraic Geom. 17, 335-340 (2008), Zbl 1138.14011 MR 2369089

Sierra, S. [2007]: A general homological Kleiman-Bertini theorem, Algebra Number Theory 3, 597-609 (2009) Zbl 1180.14048

Peskine, C., Szpiro, L. [1973]: Dimension projective finie et cohomologie locale. Applications à démonstration de conjectures de M. Auslander, H. Bass et A. Grothendieck. Inst. Hautes Études Sci. Publ. Math. 42, 47-119 (1973) Zbl 0268.13008 MR 0374130 